\title{
Akkermansia, a Possible Microbial Marker for Poor Glycemic Control in Qataris Children Consuming Arabic Diet-A Pilot Study on Pediatric T1DM in Qatar
}

\author{
Arun Prasath Lakshmanan ${ }^{1, * \mathbb{C}}$, Amira Kohil ${ }^{2}$, Farah El Assadi ${ }^{3}$, Sara Al Zaidan ${ }^{1}$, Shaikha Al Abduljabbar ${ }^{1}$, \\ Dhinoth Kumar Bangarusamy ${ }^{1}$, Fawziya Al Khalaf ${ }^{4}$, Goran Petrovski ${ }^{4}$ and Annalisa Terranegra ${ }^{1}$ (D) \\ 1 Research Department, Sidra Medicine, Doha P.O. Box 26999, Qatar; szaidan@sidra.org (S.A.Z.); \\ salabduljabbar@sidra.org (S.A.A.); dkbangarusamy@sidra.org (D.K.B.); aterranegra@sidra.org (A.T.) \\ 2 Department of Biomedical Sciences, College of Health Sciences, QU Health, Qatar University, \\ Doha P.O. Box 2713, Qatar; ak1404654@student.qu.edu.qa \\ 3 College of Health and Life Sciences, Hamad Bin Khalifa University, Doha P.O. Box 34110, Qatar; \\ felassadi@hbku.edu.qa \\ 4 Endocrinology Clinic, Sidra Medicine, Doha P.O. Box 26999, Qatar; falkhalaf@sidra.org (F.A.K.); \\ gpetrovski@sidra.org (G.P.) \\ * Correspondence: alakshmanan@sidra.org; Tel.: +974-4003-7414
}

Citation: Lakshmanan, A.P.; Kohil, A.; El Assadi, F.; Al Zaidan, S.; Al Abduljabbar, S.; Bangarusamy, D.K.; Al Khalaf, F.; Petrovski, G.; Terranegra, A. Akkermansia, a Possible Microbial Marker for Poor Glycemic Control in Qataris Children Consuming Arabic Diet-A Pilot Study on Pediatric T1DM in Qatar. Nutrients 2021, 13, 836. https:// doi.org/10.3390/nu13030836

Academic Editor: Lynnette Ferguson

Received: 10 February 2021

Accepted: 24 February 2021

Published: 4 March 2021

Publisher's Note: MDPI stays neutral with regard to jurisdictional claims in published maps and institutional affiliations.

Copyright: (c) 2021 by the authors. Licensee MDPI, Basel, Switzerland. This article is an open access article distributed under the terms and conditions of the Creative Commons Attribution (CC BY) license (https:// creativecommons.org/licenses/by/ $4.0 /)$.
Abstract: In Qatar, Type 1 Diabetes mellitus (T1DM) is one of the most prevalent disorders. This study aimed to explore the gut microbiome's relation to the continuous subcutaneous insulin infusion (CSII) therapy, dietary habits, and the HbA1c level in the pediatric T1DM subjects in Qatar. We recruited 28 T1DM subjects with an average age of $10.5 \pm 3.53$ years. The stool sample was used to measure microbial composition by $16 \mathrm{~s}$ rDNA sequencing method. The results have revealed that the subjects who had undergone CSII therapy had increased microbial diversity and genus Akkermansia was significantly enriched in the subjects without CSII therapy. Moreover, genus Akkermansia was higher in the subjects with poor glycemic control $(\mathrm{HbA} 1 \mathrm{c}>7.5 \%)$. When we classified the subjects based on dietary patterns and nationality, Akkermansia was significantly enriched in Qataris subjects without the CSII therapy consuming Arabic diet than expatriates living in Qatar and eating a Western/mixed diet. Thus, this pilot study showed that abundance of Akkermansia is dependent on the Arabic diet only in poorly controlled Qataris T1DM patients, opening new routes to personalized treatment for T1DM in Qataris pediatric subjects. Further comprehensive studies on the relation between the Arabic diet, ethnicity, and Akkermansia are warranted to confirm this preliminary finding.

Keywords: Akkermansia; T1DM; Arabic diet; ethnicity; HbA1c; CSII therapy

\section{Introduction}

Type 1 diabetes mellitus (T1DM) is a metabolic disorder, and it is caused by the autoimmune destruction of pancreatic beta cells resulting in insulin deficiency. T1DM affects all age groups irrespective of gender. Based on the International Diabetes Federation (IDF) Diabetes Atlas, the incidence of T1DM continues to increase worldwide, with approximately one million cases presented annually [1], and the diabetic prevalent rate in Qatar is around $17 \%$ [2]. T1DM is associated with various other complications, such as severe hypoglycemia, ketoacidosis, diabetic retinopathy, nephropathy, and cardiovascular complications [3]. Despite the severity and the incidence of the disease, the etiopathogenesis of T1DM is still not fully understood, involving a complex interaction between environmental and genetic factors [4].

In managing T1DM patients, the therapeutic goal is to manage glucose control, which is accomplished by different treatments, such as insulin therapy and medical nutrition therapy. Insulin therapy (basal-bolus regimen) is one of the recommended approaches in 
managing patients with T1DM. It is also known as multiple daily injection therapy. Longacting basal insulin and prandial insulin (rapid-acting) are given for food and corrections as multiple injections at different time intervals throughout the day [5]. The basal insulin dose is based on body weight and insulin sensitivity, whereas the prandial insulin dose is based on carbohydrate intake [6,7]. Basal-bolus therapy has improved glycemic control and reduced the $\mathrm{HbA1c}$ level $(<7 \%)$ in T1DM patients [8]. Insulin pump therapy (also known as Continuous Subcutaneous Insulin Infusion-CSII) involves using an insulin pump device that provides a steady insulin level to the patient. It is considered a common approach used in the out-patient treatment of T1DM [5]. One study showed that episodes of severe hypoglycemia or hyperglycemia were less common in patients under insulin pump treatment [9]. Another study demonstrated that CSII therapy is safe and effective in children and young adults with T1DM. Treatment with CSII therapy has significantly decreased $\mathrm{HbA} 1 \mathrm{c}$ levels [10].

There is a strong link between gut microbiota dysbiosis and the development of T1DM, as shown by various animal and human studies. The T1DM patients showed a less-diverse microbiome, with enhanced Bacteroides' production and reduced level of Faecalibacterium prausnitzii and Lactobacillus [11,12]. Various studies have demonstrated that gut microbial dysbiosis and enhanced gut permeability [13] result in beta cell damage and autoimmune activation, contributing to the pathogenesis of T1DM [14,15]. Animal studies have shown that insulin deficiency was associated with the bactericidal dysfunction of Paneth cells, which can alter the intestinal flora [16]. Another approach of T1DM treatment is the mesenchymal stem cell transplantation, which has been reported to deplete the diabetic gut microbiota resistance [17]. However, no clear evidence is available about gut microbiota's role in affecting the response to the T1DM treatments. For type 2 diabetes mellitus (T2DM), evidence suggests an association between metformin, a widely used anti-diabetic drug, and the gut microbiome, resulting in improved glycemic control. However, the same study showed an insignificant change in microbial diversity between the metformin and the placebo group. Only the acetate, one of the short-chain fatty acids (SCFAs), was significantly different between both groups.

In contrast, butyrate and propinoate showed no statistical difference [18,19]. Furthermore, numerous studies have pointed out that diet, geographical location, and ethnicity play a significant role in shaping individuals' gut microbiota [20]. However, the association between T1DM treatment and gut microbiota is yet to be demonstrated. No previous study investigated the association between T1DM treatment and gut microbiota in Qatar's pediatric T1DM population. In this pilot study, we aimed to understand the relationship between the gut microbiota and CSII therapy, evaluating the effect of external factors, such as the diet and nationality, in pediatric T1DM subjects living in Qatar.

\section{Materials and Methods}

\subsection{Recruitment and Sample Collection}

A pilot study recruiting 28 T1DM pediatric patients was conducted at Sidra Medicine hospital in Qatar and approved by the local IRB committee (IRB approval no \#1500755). The subjects were approached and introduced to the study after their medical appointment in Sidra's endocrinology clinic. Study subjects were selected based on the following criteria: being between the age of 6-12 years old, had no medical condition other than T1DM, a disease onset of more than one year, and no history of antibiotic treatment in the previous three months. The subjects and their parents, who agreed to participate in this study, signed parental consent and child assent forms. The physician collected the subject's clinical information, such as medication, family history of diabetes, insulin treatment, CSII therapy, and diabetes duration during the visit. Also, the dietary intake of the patients was determined by a $24 \mathrm{~h}$ food recall during the interview. Anthropometric measurements were also collected, including body weight, height, and the BMI percentile was computed according to age and gender. Stool samples were collected using the OMNIgene®stool 
collection kit (DNA Genotek, Kanata, ON, Canada ) and stored at -80 degree Celsius until further use.

\subsection{Bacterial DNA Extraction from Fecal Samples}

Bacterial DNA extraction was performed using QIAamp®Fast DNA Stool Mini kit (Qiagen, Germantown, MD, USA) according to the manufacturer's instructions. The quantity and quality of the extracted DNA were checked by using NanoDrop One (ThermoFisher Scientific, Waltham, MA, USA).

\subsection{SrDNA Library Preparation}

The library preparation and the sequencing steps were performed according to the manufacturer's instructions (MiSeq system, Illumina, San Diego, CA, USA). Briefly, Illumina primers were obtained to target V3-V4 regions of the $16 \mathrm{~S}$ rDNA gene. The PCR amplifications were carried out under the following conditions: initial at $95^{\circ} \mathrm{C}$ for $3 \mathrm{~min}$, 25 cycles at $95{ }^{\circ} \mathrm{C}$ for $30 \mathrm{~s}, 55{ }^{\circ} \mathrm{C}$ for $30 \mathrm{~s}, 72{ }^{\circ} \mathrm{C}$ for $30 \mathrm{~s}$, with a final step at $72{ }^{\circ} \mathrm{C}$ for $5 \mathrm{~min}$. Then, the secondary amplification (index PCR) was performed using Nextera XT Index Kit (Illumina, San Diego, CA, USA), where dual indices (i7 and i5 indexing primers) and Illumina sequencing adapters (P5 and P7) were added to each sample in the 96-well microplate. The PCR conditions are the same as the one previously mentioned, except the number of amplification cycles is set to 8 . The quality of the PCR products was assessed by $2 \%$ agarose gel electrophoresis. The library size was detected using Agilent High sensitivity kit (Agilent Technologies, Santa Clara, CA, USA), Agilent 200 Bioanalyzer technology, and quantified using the Qubit dsDNA HS assay kit (ThermoFisher Scientific, Waltham, MA, USA). Finally, the normalized libraries were pooled by mixing $5 \mu \mathrm{L}$ of the diluted DNA of each library with unique indices in one tube for sequencing. The pooled library and Phix control were denatured using $0.2 \mathrm{~N} \mathrm{NaOH}$ as per the manufacturer's protocol. Finally, the sample was sequenced using the Miseq reagent v3 kit-600 cycles (Illumina, San Diego, CA, USA) according to the manufacturer's instructions. Base-calling was directly carried out on the MiSeq.

The raw data were demultiplexed using MiSeq Reporter on Illumina Miseq. PEAR tool was used to merge both forward and reverse end sequences for each sample [21], and the reads with a high-quality score of 30 and above were selected using the Trimmomatic tool [22]. FASTQ files were converted into FASTA files using QIIME v1.9.0 (Quantitative Insights Into Microbial Ecology) pipeline [23]. Operational taxonomic units (OTUs) were obtained by aligning the sequence against the Greengenes database (gg_13_08) with a confidence threshold of $97 \%$ [24].

\subsection{Data Analysis}

\subsubsection{Clinical Data}

The clinical data obtained from the study subjects include gender, nationality, anthropometric measurements (height, weight, BMI percentiles), HbA1c, diabetes duration, and CSII therapy. The two-tails unpaired $t$-test was used to define group differences. $p<0.05$ was considered statistically significant.

\subsubsection{Dietary Data}

The dietary data obtained from $24 \mathrm{~h}$ food recall was used to evaluate the dietary patterns. The first analysis classified the patients into two groups based on the primary type of consumed foods: Arabic diet, consuming food and recipes from the Arabic culinary tradition; mixed diet consuming mainly or exclusively Western-like food.

\subsubsection{Microbiome Data}

Alpha diversity and beta diversity analysis were performed using the R package [25-27]. Linear discriminant analysis effect size (LEfSe) analysis was used to find out microbial biomarkers among the different groups with the cutoff value of LDA >2.0 [28]. 


\section{Results}

\subsection{Study Population and Dietary Habits}

We recruited 28 T1DM patients in the age range of $10.5 \pm 3.5$ years, in which half of the study subjects were Qatari Nationals, and half of the expatriates lived in Qatar. Females accounted for $35.7 \%$ of the study subjects, and males accounted for $64.2 \%$, with a mean BMI percentile of $57.59 \pm 29.92$ in the range of the normal weight. The HbA1c average level and the diabetes duration for the total study subjects were $9.75 \pm 1.62 \%$ and $8 \pm 4.24$ years, respectively. Out of the 28 participants, only 11 subjects had undergone CSII therapy. Based on the dietary pattern, $56 \%$ of the study subjects consumed an Arabic diet, whereas $42.8 \%$ consumed a mixed Western-like diet. Most of the Qatari Nationals (62.5\%) consumed the Arabic diet, compared to the expatriates consuming mostly a mixed Western-like diet (66\%). The baseline characteristics of the study subjects are shown in Table 1.

Table 1. General characteristics of the study participants.

\begin{tabular}{|c|c|}
\hline Parameters & T1DM Patients \\
\hline Number of subjects & 28 \\
\hline Age in years & $10.50 \pm 3.53$ \\
\hline \multicolumn{2}{|l|}{ Gender } \\
\hline Female & $10(39.3 \%)$ \\
\hline Male & $18(60.7 \%)$ \\
\hline \multicolumn{2}{|l|}{ Dietary pattern } \\
\hline Arabic diet & $16(57 \%)$ \\
\hline Mixed Western-like diet & $12(42.8 \%)$ \\
\hline \multicolumn{2}{|l|}{ Nationality } \\
\hline Qatari & $14(50 \%)($ Arabic diet $=10$, mixed diet $=4)$ \\
\hline Expatriate & $14(50 \%)($ Arabic diet $=6$, mixed diet $=8)$ \\
\hline $\mathrm{HbA1c}(\%)$ & $9.75 \pm 1.62$ \\
\hline$<7.5 \%$ & $10(35.7 \%)($ Arabic diet $=06$, mixed diet $=04)$ \\
\hline$>7.5 \%$ & $18(64.3 \%)($ Arabic diet $=10$, mixed diet $=08)$ \\
\hline \multicolumn{2}{|l|}{ CSII } \\
\hline Yes & $11(39.3 \%)($ Arabic diet $=04$, mixed diet $=07)$ \\
\hline No & $17(60.7 \%)($ Arabic diet $=12$, mixed diet $=05)$ \\
\hline Diabetes Duration (years) & $8.00 \pm 4.24$ \\
\hline BMI Percentile & $57.59 \pm 29.92$ \\
\hline
\end{tabular}

Note-The values in the table are represented as mean \pm SD and in percentage, wherever applicable. CSII: Continuous Subcutaneous Insulin Infusion; HbA1c: Hemoglobin A1c.

\subsection{Effect of CSII Therapy and HbA1c Level on the Gut Microbiome Composition in the T1DM Subjects}

We have measured the relative abundance of gut microbiota in our T1DM study subjects. Alpha diversity analysis has revealed that the microbial abundance level (measured by Simpson) was higher in the CSII group than the non-CSII group. Still, there was no statistically significant difference in the genus richness (measured by Observed and Chao1) between these two groups (Figure 1a). Moreover, beta diversity analysis has shown no statistical difference between these two groups (Figure 1b). The significant bacteria at the phyla level were Bacteroidetes, Firmicutes, Actinobacteria, Proteobacteria, and Verrucomicrobia (Supplementary Figure S1). We then evaluated CSII therapy's effect and the HbA1c level on the gut microbial composition in the T1DM subjects. However, the identification of gut microbial markers using the LEfSe analysis has demonstrated that the phylum Verrucomicrobia and particularly the genus Akkermansia were significantly enriched in the T1DM patients without CSII therapy. 
(a)

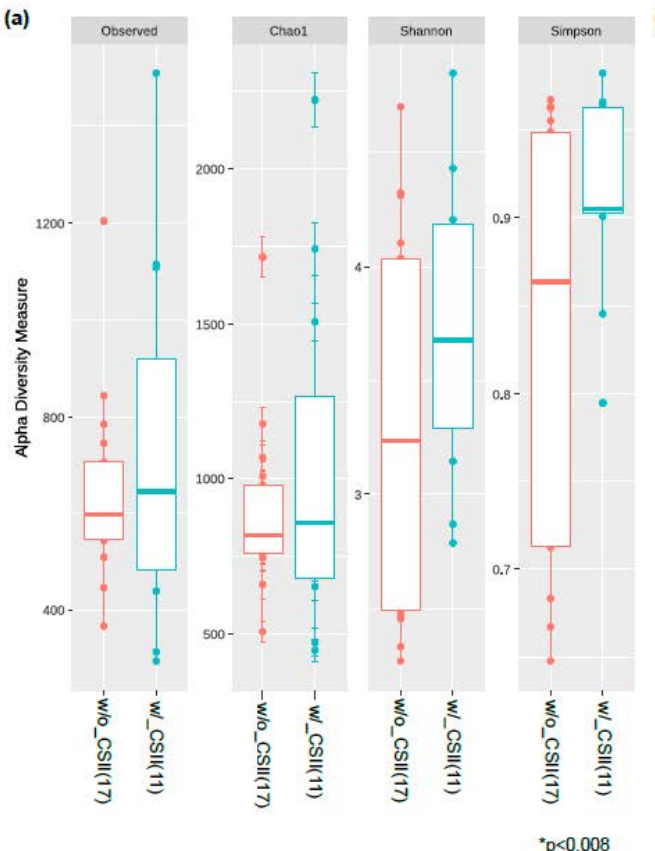

(b)

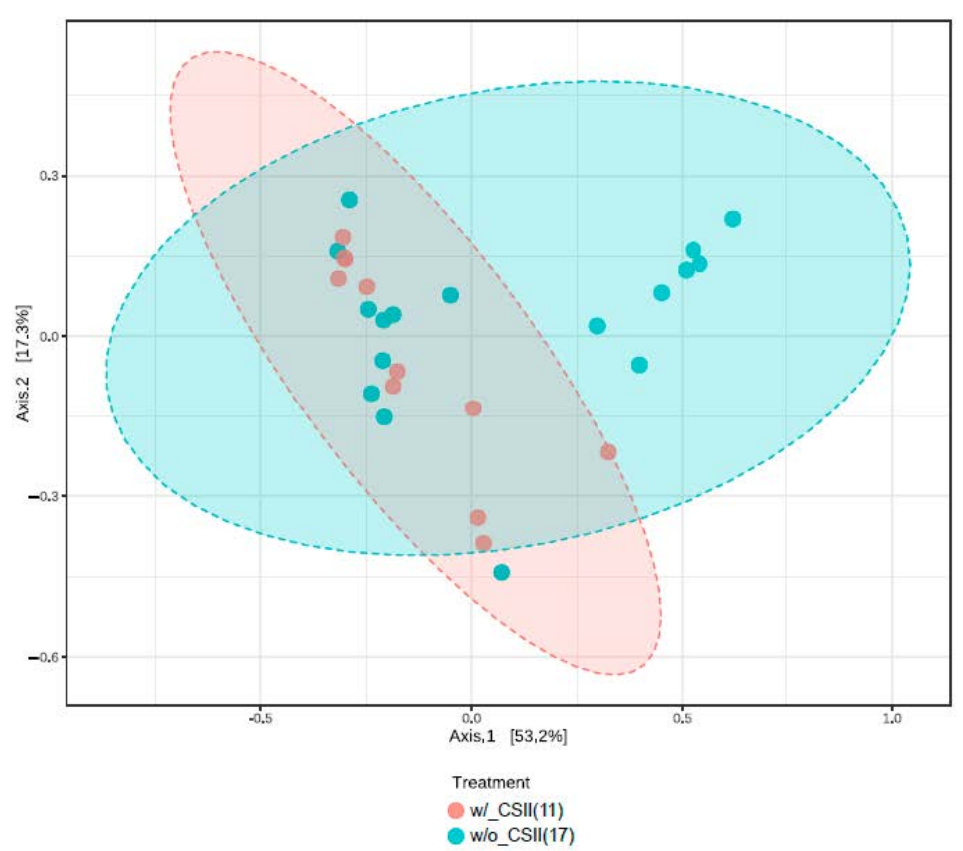

Figure 1. Effect of CSII therapy on the gut microbiome composition in the T1DM subjects. (a) The gut microbial diversity index measured by alpha diversity revealed that the subjects who had CSII therapy had higher microbial abundance (only in Simpson index). (b) There were no microbial dissimilarities (beta-diversity) in subjects with or without the CSII therapy. $\beta$-Diversity was visualized using Principle co-ordinations generated with the Bray-Curtis distance metric using QIIME. Analysis of group similarity (ANOSIM) was measured between categories included in this study using 1000 permutations, ${ }^{*} p<$ 0.05. (b) Note: g UC, genus_unclassified; w/_CSII, with CSII therapy; w/o_CSII, without CSII therapy.

In contrast, the family Christensenellaceae, order Neisseriales, and genus Klebsiella, Escherichia, Pseudobutyrivibrio, and Aggregatibacter were increased in subjects who had undergone CSII therapy (Figure 2a). Interestingly, Akkermansia was found to be enriched in the subjects with poor glycemic control $(>7.5 \%)$ than the subjects with better glycemic control $(<7.5 \%)$ (Figure $2 b)$.

\subsection{Impact of Diet on the Abundance of the Genus Akkermansia in the Poorly Controlled T1DM Subjects}

We investigated the impact of diet on the abundance of the phylum Verrucomicrobia and the genus Akkermansia in the T1DM subjects with poor glycemic control and the CSII therapy. Phylum Verrucomicrobia and the genus Akkermansia significantly enriched in the Arabic-diet-consuming subjects without the CSII therapy (Figure 3a). In addition to this, Akkermansia level was higher only in the poorly controlled subjects having a high level of $\mathrm{HbA1c}(>7.5 \%)$ and consuming the Arabic diet (Figure 3b). Interestingly, subjects with a high level of $\mathrm{HbA1c}$ had an elevated level of the genus Ruminococcus (Figure 2b), and the subjects consuming the Arabic diet and with a high level of $\mathrm{HbA} 1 \mathrm{c}$ also showed an elevated level of Ruminococcus (Figure 3b).

\subsection{Impact of Nationality on the Abundance of the Genus Akkermansia in the Poorly Controlled T1DM Subjects}

Interestingly, we have found that the phylum Verrucomicrobia and genus Akkermansia were enriched only in the Qataris subjects without the CSII therapy, but not in the expatriates (Figure 4a). When we classified the subjects with the combination of HbA1c and nationality, surprisingly, we did not see an enrichment of Akkermansia in any group (Figure 4b). 
(a)

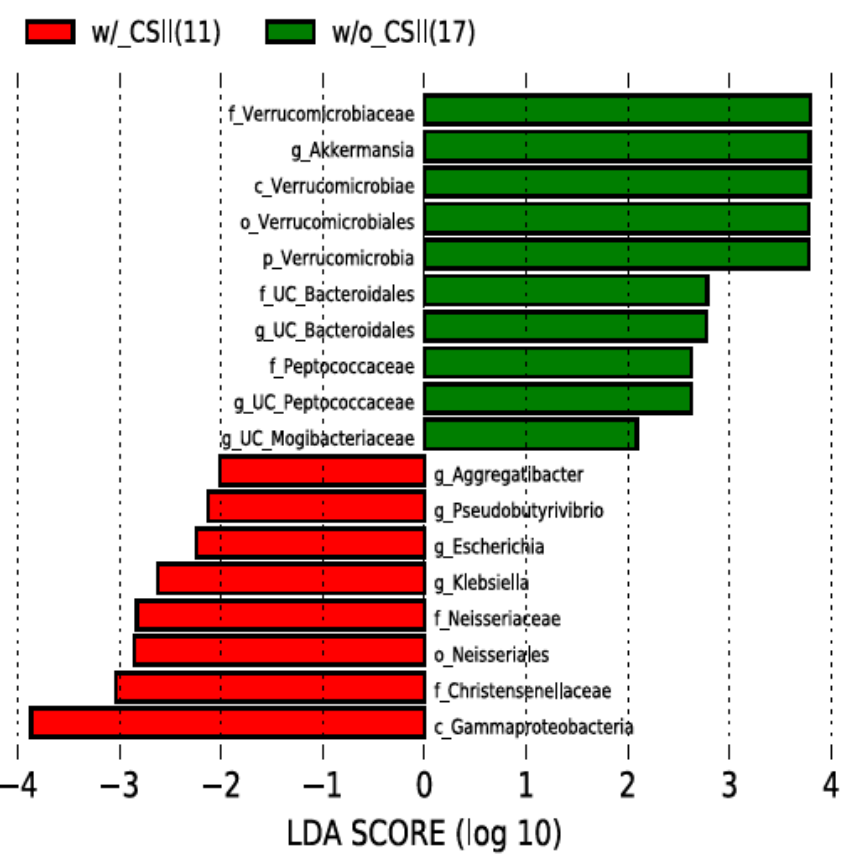

(b)

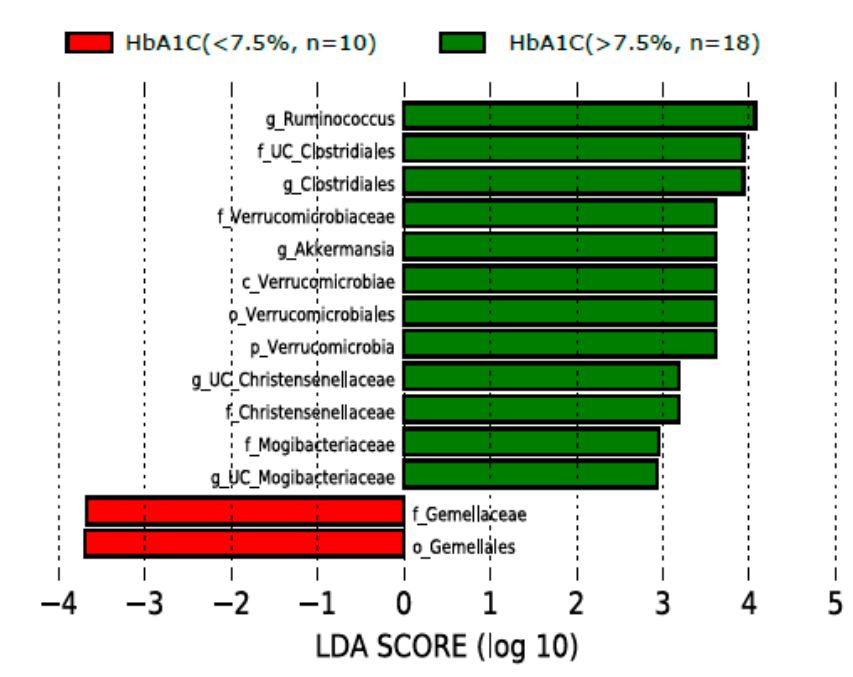

Figure 2. Effect of CSII therapy and HbA1C level on the gut microbiome composition in the T1DM subjects. The LEfSe analysis showed (a) significant enrichment of Akkermansia in the subjects without CSII therapy and (b) significant enrichment of Akkermansia in the subjects with $\mathrm{HbA1c}$ level of more than $>7.5 \%$ and $<7.5 \%$ LDA cutoff value $>2.0$. Note: g_UC, genus_unclassified.

3.5. Influence of Arabic Diet in Qataris Subjects on the Abundance of the Genus Akkermansia in the T1DM Subjects

Here, we have intended to explain the role of Qataris nationality and the Arabic diet on the abundance of Akkermansia in the study population. Interestingly, we have found that Qataris pediatric T1DM subjects had elevated levels of Akkermansia compared to their counterpart (Figure 5a). Surprisingly, we did not find an enriched Akkermansia level when we classified the subjects based only on the diet pattern (Figure $5 b$ ).

Finally, when we analyzed the data based on both nationality and the dietary pattern, only Qataris pediatric T1DM subjects who consumed the Arabic diet had shown a higher abundance of Akkermansia among the other groups (Figure 6). 
(a)

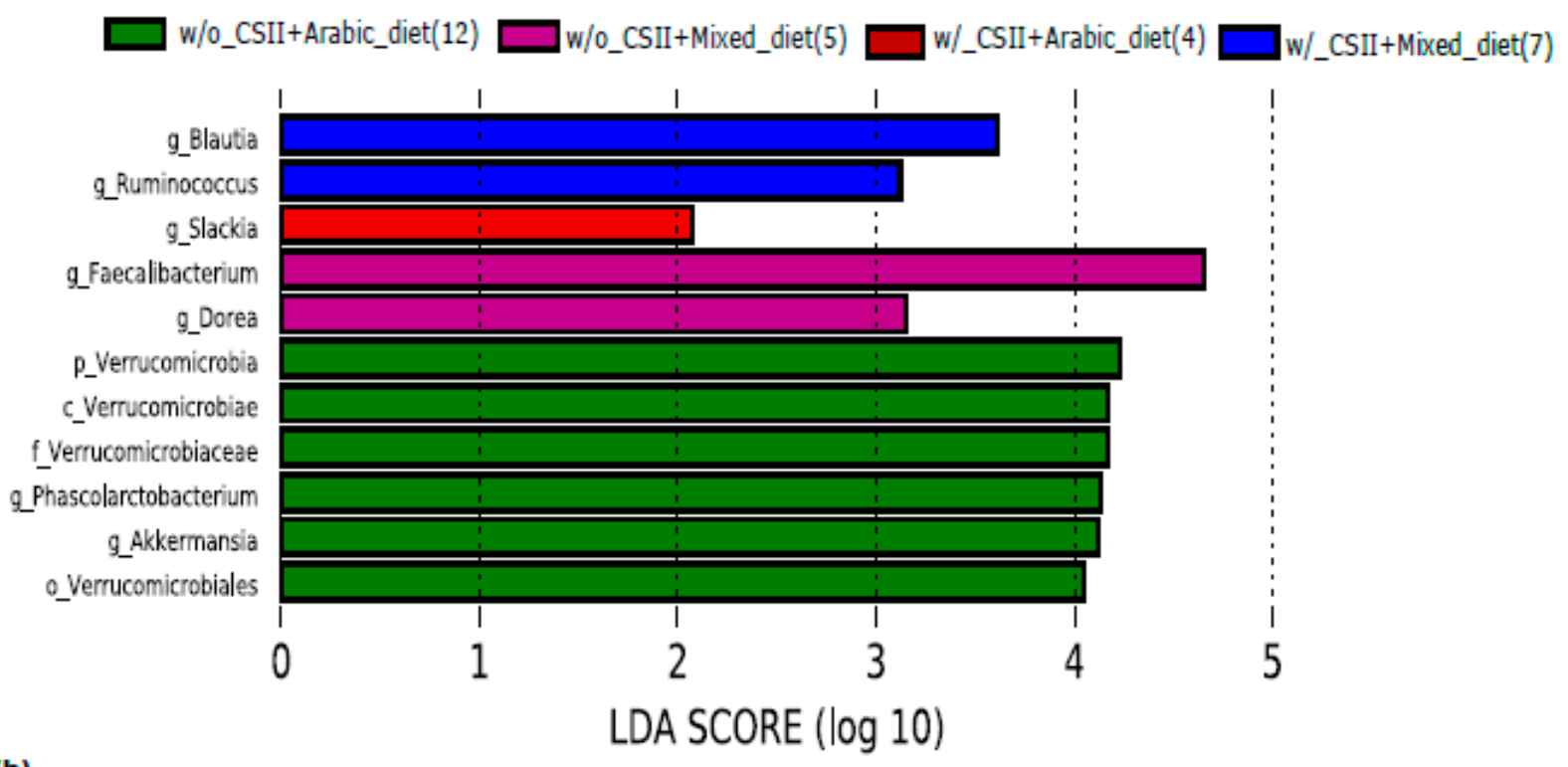

(b)

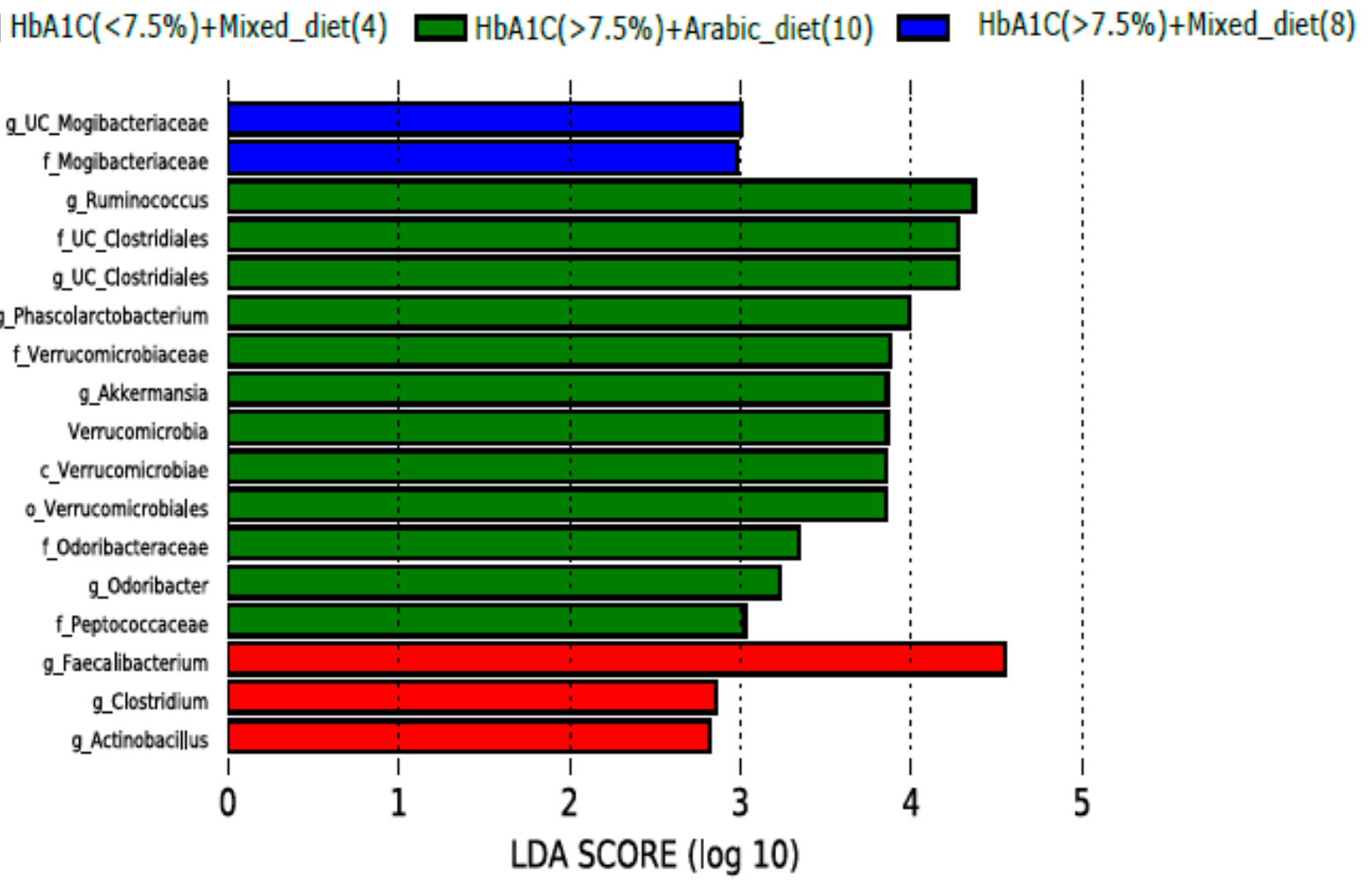

Figure 3. Impact of CSII therapy and diet on genus Akkermansia in poorly controlled T1DM subjects. (a,b) LEfSe analysis revealed that Akkermansia was enriched only in the subjects without CSII therapy treatment and poor glycemic control who consumed the Arabic diet. LDA cutoff value >2.0; Note: g_UC, genus_unclassified; w/_CSII, with CSII therapy; w/o_CSII, without CSII therapy. 
(a)

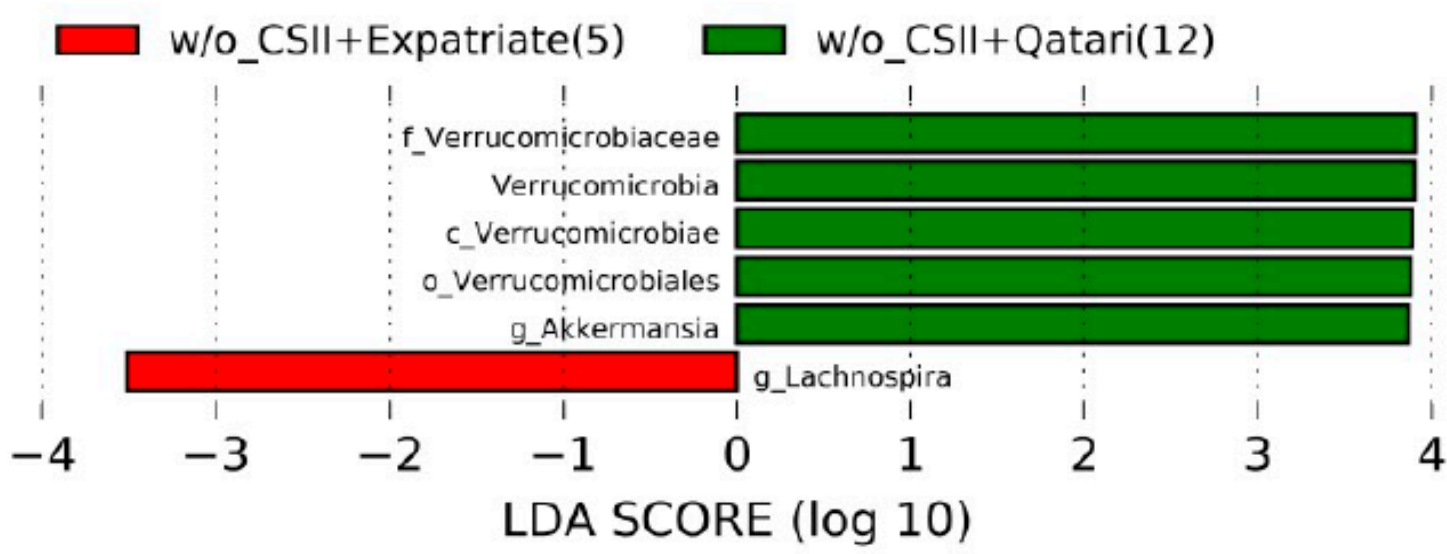

(b)

$\square<7.5+$ Expatriate(8) $\square>7.5+$ Expatriate(6) $\square>7.5+$ Qatari(12)

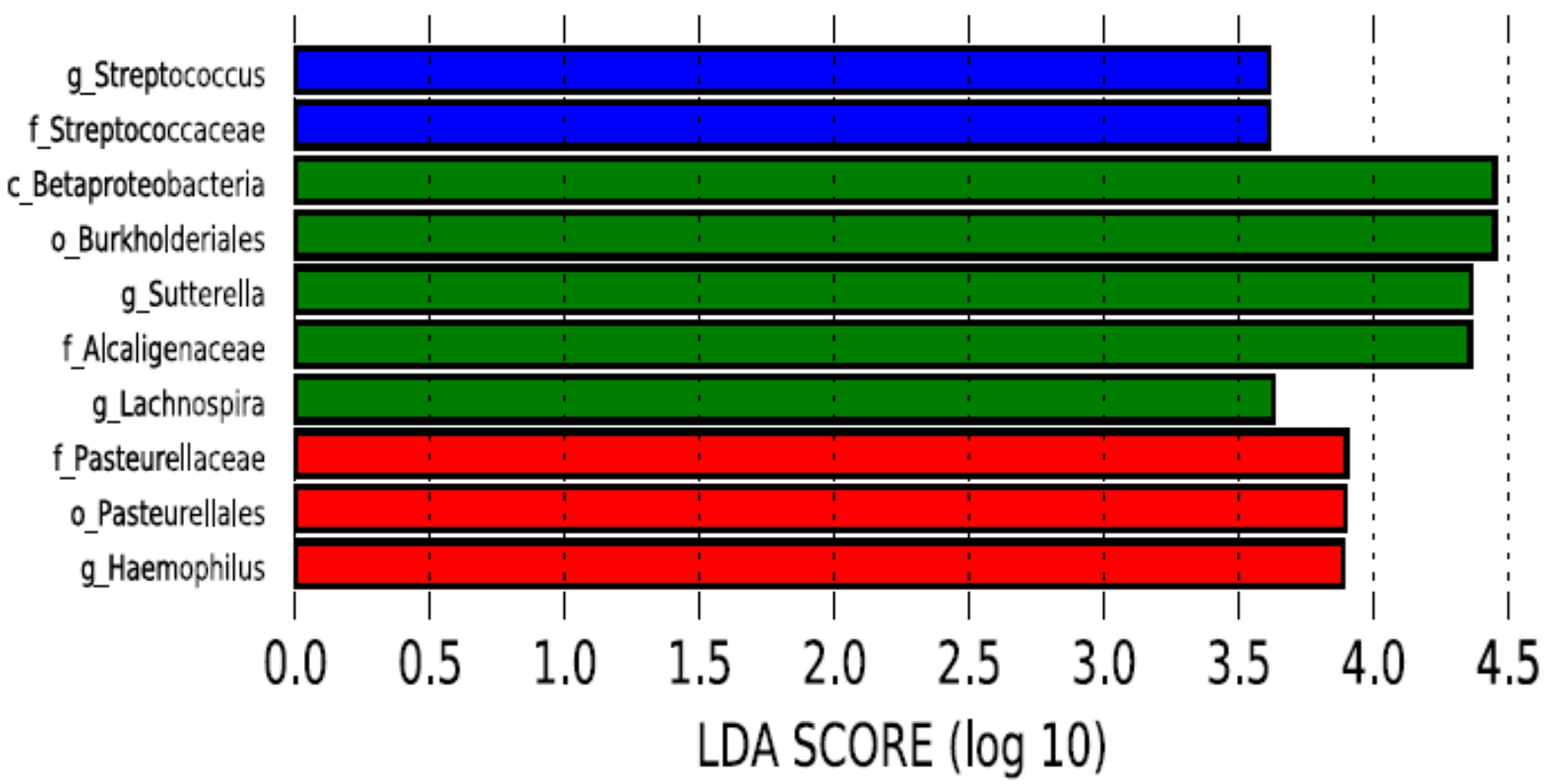

Figure 4. Impact of CSII therapy and nationality on genus Akkermansia in poorly controlled T1DM subjects. (a) Akkermansia was enriched only in the Qataris T1DM subjects without CSII therapy and (b) Akkermansia disappeared analyzing HbA1c levels according to nationality. LDA cutoff value > 2.0; Note: g_UC, genus_unclassified; w/_CSII, with CSII therapy; w/o_CSII, without CSII therapy. 
(a)

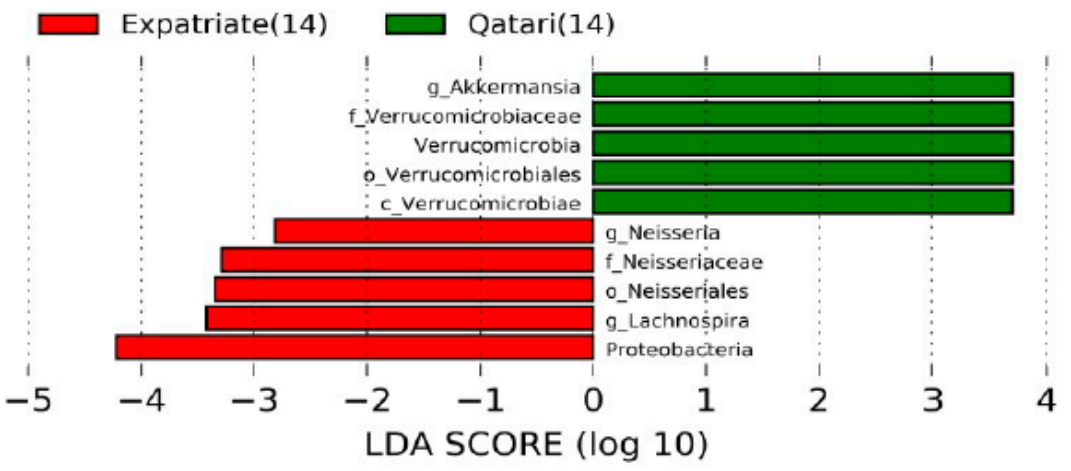

(b)

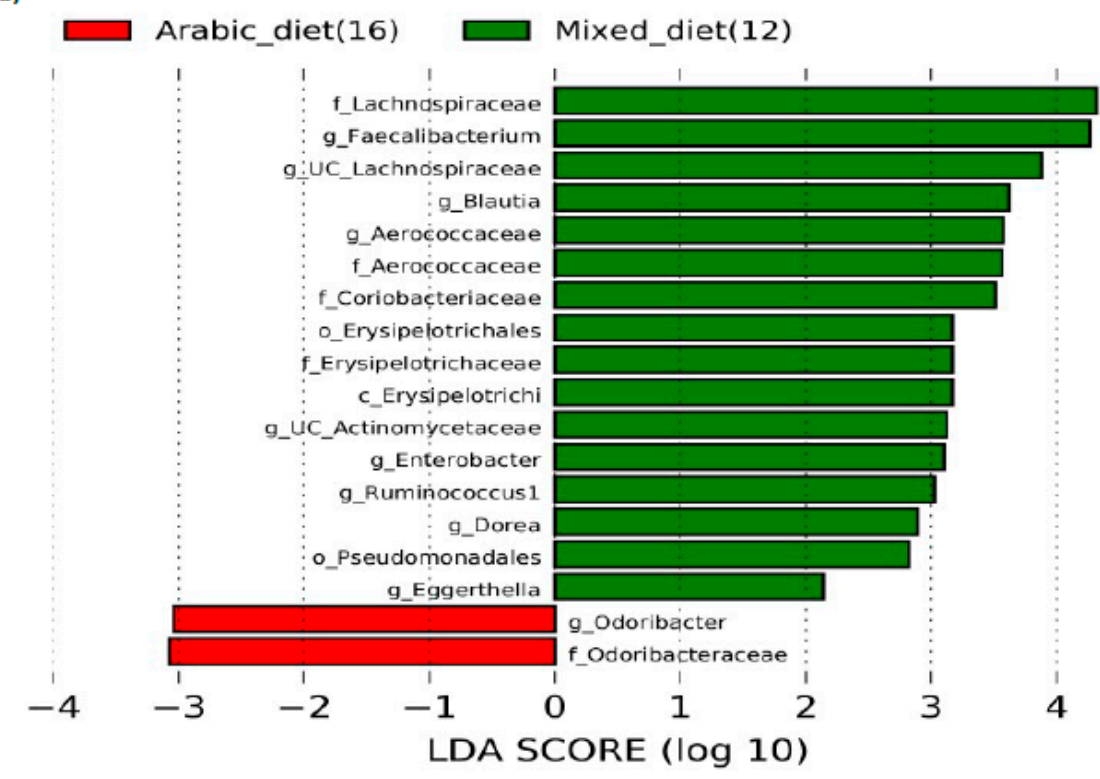

Figure 5. Impact of diet and nationality on genus Akkermansia in T1DM subjects. LEfSe analysis has revealed that (a) the Akkermansia was only enriched in the Qataris T1DM subjects, and (b) Akkermansia was not significantly different between the Arabic diet and mixed diet consumed subjects. LDA cutoff value $>2.0$ g_UC represents genus_unclassified.

Arabic_diet+expatriate(6) $\square$ Arabic_diet+Qatari(6)

Mixed_diet+expatriate(8)

Mixed_diet+Qatari(4

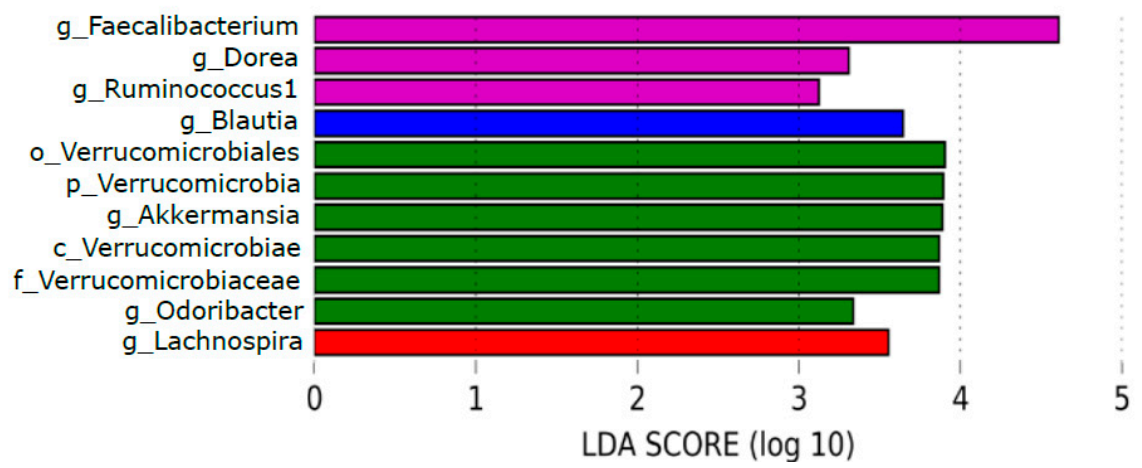

Figure 6. Impact of combination of diet and nationality on genus Akkermansia in T1DM subjects. LEfSe analysis has revealed that the Akkermansia was only enriched in the Qataris T1DM subjects, particularly in Qataris who consumed the Arabic diet. LDA cutoff value $>2.0$ g_UC represents genus_unclassified. 


\section{Discussion}

In this pilot study, we aimed to identify the effect of CSII therapy and the HbA1c level on the gut microbiome composition and the impact of diet and nationality in the T1DM pediatric subjects living in Qatar. To the best of our knowledge, this is the first study that showed the genus Akkermansia as a potential microbial marker of insulin treatment response. Its abundance is affected by the nationality and the diet in the pediatric T1DM subjects living in Qatar. It has been well established that both the T1DM and gut microbiome regulate each other in humans and animal models [29-32]. The relationship between these two factors is complex in nature. Studies have indicated that one of the earliest indications for dysbiosis in T1DM is the Bacteroidetes/Firmicutes ratio changes and the reduced diversity index in the pediatric T1DM subjects [33,34]. Interestingly, in this study, we did not find any significant differences in the Bacteroidetes/Firmicutes ratio.

The classical treatment strategy for the maintenance of normoglycemia in the T1DM subjects is the administration of insulin. Insulin can be administered via single-dose injection or multiple-dose injections, depending on the insulin dose requirement. Insulin dose is determined based on the body weight and the carbohydrate coverage, i.e., insulinto-carbohydrate ratio $[35,36]$. In addition to this, CSII therapy in children is getting good acceptance by the healthcare providers. The SWEET registry cohort data has shown that the children who underwent CSII therapy had achieved reasonable metabolic control compared to the children without CSII therapy [37]. In this study, initially, we aimed to find the effect of CSII on the gut microbiome of pediatric T1DM subjects, and the results have shown that the genus Akkermansia was significantly enriched in the subjects without the CSII therapy. Moreover, genus abundance measured by the Simpson method has shown that the subjects with CSII therapy had higher abundance than their counterpart. We aimed to measure the microbiome composition in poorly controlled patients ( $\mathrm{HbA} 1 \mathrm{c}$ level > 7.5\%). $\mathrm{HbA1c}$ test estimates a patient's three-month average blood glucose level and is a widely accepted measure in diabetic subjects to evaluate their glycemic control [38]. Surprisingly, Akkermansia was significantly enriched in the poorly controlled group compared to the controlled group. Indeed, it is fascinating because Akkermansia muciniphila (A. muciniphila), a mucin degrading bacterium, has been widely reported to play a significant role in promoting the gut barrier function, epithelial cell integrity, and enhancement of transepithelial resistance through various mechanistic pathways such as the expression of genes related to the immune response, normalizing the metabolic endotoxemia and lipid metabolism [39-44]. A. muciniphila has been found to mediate the metformin's beneficial effects, such as the regulation of glucose metabolism via the SCFAs production in human and mice studies $[45,46]$. Few contrary reports suggest that the $A$. muciniphila level was elevated during the progression of chronic kidney diseases (CKD) [47]. The Bio-Breeding Diabetic Prone (BBdp) rats have shown that mucin reduction increases the susceptibility to T1DM [48]. In addition to this, administration of $A$. muciniphila grown-up in a mucin-less environment has a significant reduction effect on the obesity in HFD-induced obesity in mice than the A. muciniphila grown-up in a mucin-rich environment. So, it is evident that the mucin content plays a pivotal role in the gut barrier function [49]. However, $A$. muciniphila in most diseases seem to play protective roles and in a few conditions like T1DM and CKD, where it might play a controversial role. More comprehensive studies are needed to clarify the exact role of $A$. muciniphila in the T1DM subjects.

Diet and environment can play a role in explaining the controversial findings around A. muciniphila. Diet is known to modulate Akkermansia level, as demonstrated in animal models where the Western high-fat diet drastically reduced the abundance of Akkermansia in Apo $\mathrm{e}^{\mathrm{e} /-}$ mice, restored only after the supplementation of A. muciniphila [50]. In agreement with this study, we have found that the Akkermansia was significantly enriched in the subjects without CSII therapy and non-controlled $\mathrm{HbA} 1 \mathrm{c}$ level ( $>7.5 \%)$ consuming the Arabic diet compared to the mixed Western-like diet. Moreover, previous evidence suggests that the abundance of A. muciniphila is positively associated with the family Ruminiococcaceae, genus Gordonibacter, and species Methanobrevibacter smithii [51,52]. In this study, we also 
found that the genus Ruminococcus was elevated along with the Akkermansia in the subjects who had high HbA1c levels and consumed the Arabic diet.

Furthermore, we evaluated the impact of nationality on the abundance of Akkermansia. Interestingly, our data revealed that only the Qatari pediatric T1DM subjects without the CSII therapy conserved the microbial profile characterized by the abundance of Akkermansia compared to the expatriate's T1DM subjects living in Qatar. It suggests that host genetic factors might be involved, as previously reported, where the host genetic background could modulate the gut microbiota composition [53,54]. A recent study on monozygotic (MZ) and dizygotic (DZ) twins has shown that MZ twins had greater microbial similarities than the DZ. The different gut microbial compositions were observed in mice with mutations in genes involved in the inflammatory pathways and diabetes compared to their wildtype counterparts $[55,56]$. To test this hypothesis, we have analyzed the gut microbial composition based on nationality. Strikingly, the results showed that the Akkermansia is significantly enriched only in the Qatari pediatric T1DM subjects, not in the expatriate's T1DM subjects. So, we cannot rule out the possibility of host genetic factors for an elevated level of Akkermansia in the pediatric T1DM subjects. When we analyzed the gut microbial composition data based solely on the dietary pattern, it has revealed no apparent difference of Akkermansia between the subjects who consumed the Arabic diet compared to the mixed Western-like diet. So, there could be a possibility that host genetic factors might have a more pronounced effect on the gut microbial modulation than the dietary factors.

Interestingly, when we classified the gut microbial abundance based on the combination of two factors, i.e., a diet with nationality, we have seen the enrichment of Akkermansia only in the Qataris T1DM subjects who consumed the Arabic diet. So, the dietary pattern is still playing its part in modulating the Akkermansia level in the Qataris pediatric T1DM subjects. Supplementary Figure S2 shows the relative abundance level of the genus Akkermansia in the subjects classified at various combinations and, moreover, when we compared the relative abundance of Akkermansia with the healthy control (HC) subjects, we have found that there was a significant increase $(p=0.0097)$ in the abundance level of genus Akkermansia in T1DM subjects Supplementary Figure S2 (j), and there could be a possibility that the occurrence of T1DM would increase the rate of mucous secretion in these pediatric subjects.

The Qataris population has a unique genetic background, as confirmed from the Qatar Genome Project (QGP) that identified $58.37 \%$ novel genetic variants in Qataris. It should be considered in interpreting the data [57]. In support of this concept, a pilot study from an Italian group suggested that the gut microbiota profiles differ among individuals depending on their region of origin [58]. In support of this concept, A large cohort study conducted by Turpin et al. (2016) has shown that the gut microbiome could have greater association with the host genetic factor [59]. Composition of gut microbiota is mainly influenced by both environmental and genetic factors [60] in T1DM. In recent times, researchers with the help of sophisticated computational analysis established that one of the main focuses in the treatment of T1DM is understanding the origin of gut microbial dysbiosis. The contribution of the host genetic factor in shaping the gut microbial composition was well demonstrated by Mullaney et al. (2018) and others using non-obese diabetic (NOD) mice animal model $[61,62]$. They showed that NOD mice carry the protective alleles of T1DM susceptibility loci, namely, major histocompatibility complex (MHC) and Idd3 and $I d d 5$, in which Idd3 and Idd5 improves the regulatory T cells (Tregs) function and immune tolerance along with the distinct gut microbial composition with an increased abundance of Akkermansia. In addition to this, environmental factors, such as diet, infection, and usage of antibiotics, can have more a profound effect on the pathogenesis of T1DM. For example, Zhang et al. (2018) demonstrated that single-course usage of tyrosine tartrate (macrolide antibiotic) accelerates the occurrence of T1DM in male NOD pups, suggesting a crucial link of antibiotics in early life [63]. On the contrary, recent clinical and pre-clinical studies suggested that not all antibiotics impact on pathogenesis of T1DM, especially in shaping the gut microbiome in early life period $[64,65]$. Furthermore, usage of vancomycin increased 
the abundance of Akkermansia and modulates the glucose metabolism in mice [66]. Finally, shaping of gut microbiota in children, particularly at a younger age might be affected with various environmental factors and genetic factors, and careful interpretation is needed for the treatment of T1DM.

\section{Conclusions}

In this pilot study, we found that the genus Akkermansia, a mucin-degrading bacterium, was significantly elevated in the Qatari pediatric T1DM subjects with poorly controlled $\mathrm{HbA1c}$ levels (>7.5\%) and consuming an Arabic diet. A combined effect of host genetic factors along with the dietary habits can explain the elevated level of Akkermansia leading to higher mucous production. Novel genetic variants found in the Qatari population [57] could contribute to an increase in the level of Akkermansia in pediatric T1DM subjects. In addition, the consumption of an Arabic diet at a younger age might play a role in modulating the level of Akkermansia, since dietary pattern at a younger age is involved in shaping the gut microbiome. So, there is a possibility that the combination of genetic and dietary factors could play significant roles in defining or shaping the gut microbiota, especially in pediatric T1DM subjects. All these hypotheses warrant a larger cohort study using Qatari pediatric subjects to better understand the interaction of host genetic factors and diet on the level of Akkermansia in pediatric T1DM subjects.

\section{Study Limitation}

Our study comes with some limitations, mainly due to the small number of subjects and the use of $24 \mathrm{~h}$ diet recalls for diet analysis that reporting a one-day diet may not represent the patients' habits. Another limit is that it is impossible to further classify the expatriates according to the country of their origin. Our study's strength is the well-selected group of patients, with a confirmed diagnosis of T1DM and a good number of subjects with CSII therapy compared with subjects using insulin injection. We tried to mitigate the uncertainties of dietary records recruiting patients in a narrow age range (6-12 years old) that exclude infants, still under breastfeeding and complimentary food and with an immature microbiome, and teenagers with a wide range of social and dietary habits. Larger studies are needed to confirm the preliminary findings of this pilot study, inclusive of detailed dietary intakes and nationality information, ideally correlated by genetic analysis.

Supplementary Materials: The following are available online at https: / www.mdpi.com/2072-6 $643 / 13 / 3 / 836 / s 1$, Figure S1. Shows the gut microbial relative abundance of study participants at phyla level. Figure S2. Shows the relative abundance of genus Akkermansia in T1DM subjects and classified into various groups.

Author Contributions: Conceptualization, G.P. and A.T.; data curation, A.P.L., S.A.Z. and S.A.A.; formal analysis, A.P.L., S.A.Z. and S.A.A.; funding acquisition, A.T.; methodology, A.P.L., D.K.B., F.A.K. and G.P.; supervision, A.T.; writing-original draft, A.P.L. and A.K.; writing-review and editing, A.P.L., F.E.A., F.A.K., G.P. and A.T. All authors have read and agreed to the published version of the manuscript.

Funding: This study was supported by the Sidra Medicine Internal Research Fund (2017) awarded to Dr. Terranegra (SDR200010).

Institutional Review Board Statement: The study was conducted according to the guidelines of the Declaration of Helsinki, and approved by the Institutional Review Board of SIDRA MEDICINE (protocol code \#1500755 and 10/12/2017).

Informed Consent Statement: Informed consent was obtained from all subjects involved in the study (IRB approval \#1500755).

Data Availability Statement: The data presented in this study can be found here in the NCBI's Bio project repository: [PRJNA693107 and PRJNA701159].

Acknowledgments: The authors would like to thank Sidra Medicine for financial support to conduct this study. 
Conflicts of Interest: The authors declare no conflict of interest.

\section{References}

1. Patterson, C.C.; Karuranga, S.; Salpea, P.; Saeedi, P.; Dahlquist, G.; Soltesz, G.; Ogle, G.D. Worldwide estimates of incidence, prevalence and mortality of type 1 diabetes in children and adolescents: Results from the International Diabetes Federation Diabetes Atlas, 9th edition. Diabetes Res. Clin. Pract. 2019, 157, 107842. [CrossRef]

2. World Health Organization. Global Report on Diabetes; World Health Organization: Geneva, Switzerland, 2016.

3. Bjornstad, P.; Snell-Bergeon, J.K.; Nadeau, K.J.; Maahs, D.M. Insulin sensitivity and complications in type 1 diabetes: New insights. World J. Diabetes 2015, 6, 8-16. [CrossRef] [PubMed]

4. Atkinson, M.A.; Eisenbarth, G.S.; Michels, A.W. Type 1 diabetes. Lancet 2014, 383, 69-82. [CrossRef]

5. Mendez, C.E.; Umpierrez, G.E. Management of Type 1 Diabetes in the Hospital Setting. Curr. Diabetes Rep. 2017, 17, 98. [CrossRef] [PubMed]

6. American Diabetes Association. 4. Comprehensive Medical Evaluation and Assessment of Comorbidities: Standards of Medical Care in Diabetes-2019. Diabetes Care 2019, 42, S34-S45. [CrossRef] [PubMed]

7. Janez, A.; Guja, C.; Mitrakou, A.; Lalic, N.; Tankova, T.; Czupryniak, L.; Tabak, A.G.; Prazny, M.; Martinka, E.; Smircic-Duvnjak, L. Insulin Therapy in Adults with Type 1 Diabetes Mellitus: A Narrative Review. Diabetes Ther. 2020, 11, 387-409. [CrossRef] [PubMed]

8. Boudiba, A.; Alarouj, M.; Li, R.; Chantelot, J.; Al-Rubeaan, K. Effectiveness and safety of basal-bolus therapy (insulin glargine+insulin glulisine) in patients with type 1 diabetes previously uncontrolled on any insulin regimen: Multinational phase-IV study. Diabetes Manag. 2017, 7, 159-169.

9. Cook, C.B.; Beer, K.A.; Seifert, K.M.; Boyle, M.E.; Mackey, P.A.; Castro, J.C. Transitioning insulin pump therapy from the outpatient to the inpatient setting: A review of 6 years' experience with 253 cases. J. Diabetes Sci. Technol 2012, 6, 995-1002. [CrossRef] [PubMed]

10. Plotnick, L.P.; Clark, L.M.; Brancati, F.L.; Erlinger, T. Safety and effectiveness of insulin pump therapy in children and adolescents with type 1 diabetes. Diabetes Care 2003, 26, 1142-1146. [CrossRef] [PubMed]

11. Vaarala, O. Human intestinal microbiota and type 1 diabetes. Curr. Diabetes Rep. 2013, 13, 601-607. [CrossRef] [PubMed]

12. Paun, A.; Yau, C.; Danska, J.S. The Influence of the Microbiome on Type 1 Diabetes. J. Immunol. 2017, 198, 590-595. [CrossRef] [PubMed]

13. Harbison, J.E.; Roth-Schulze, A.J.; Giles, L.C.; Tran, C.D.; Ngui, K.M.; Penno, M.A.; Thomson, R.L.; Wentworth, J.M.; Colman, P.G.; Craig, M.E.; et al. Gut microbiome dysbiosis and increased intestinal permeability in children with islet autoimmunity and type 1 diabetes: A prospective cohort study. Pediatr. Diabetes 2019, 20, 574-583. [CrossRef] [PubMed]

14. Fassatoui, M.; Lopez-Siles, M.; Diaz-Rizzolo, D.A.; Jmel, H.; Naouali, C.; Abdessalem, G.; Chikhaoui, A.; Nadal, B.; Jamoussi, H.; Abid, A.; et al. Gut microbiota imbalances in Tunisian participants with type 1 and type 2 diabetes mellitus. Biosci. Rep. 2019, 39, BSR20182348. [CrossRef] [PubMed]

15. Durazzo, M.; Ferro, A.; Gruden, G. Gastrointestinal Microbiota and Type 1 Diabetes Mellitus: The State of Art. J. Clin. Med. 2019, 8, 1843. [CrossRef] [PubMed]

16. Yu, T.; Yang, H.S.; Lu, X.J.; Xia, Z.S.; Ouyang, H.; Shan, T.D.; Huang, C.Z.; Chen, Q.K. Association of Bactericidal Dysfunction of Paneth Cells in Streptozocin-Induced Diabetic Mice with Insulin Deficiency. Med. Sci. Monit. 2016, 22, 3062-3072. [CrossRef]

17. Lv, W.; Graves, D.T.; He, L.; Shi, Y.; Deng, X.; Zhao, Y.; Dong, X.; Ren, Y.; Liu, X.; Xiao, E.; et al. Depletion of the diabetic gut microbiota resistance enhances stem cells therapy in type 1 diabetes mellitus. Theranostics 2020, 10, 6500-6516. [CrossRef] [PubMed]

18. Ejtahed, H.S.; Tito, R.Y.; Siadat, S.D.; Hasani-Ranjbar, S.; Hoseini-Tavassol, Z.; Rymenans, L.; Verbeke, K.; Soroush, A.R.; Raes, J.; Larijani, B. Metformin induces weight loss associated with gut microbiota alteration in non-diabetic obese women: A randomized double-blind clinical trial. Eur. J. Endocrinol. 2019, 180, 165-176. [CrossRef] [PubMed]

19. Adeshirlarijaney, A.; Gewirtz, A.T. Considering gut microbiota in treatment of type 2 diabetes mellitus. Gut Microbes 2020, 11, 253-264. [CrossRef]

20. Senghor, B.; Sokhna, C.; Ruimy, R.; Lagier, J.-C. Gut microbiota diversity according to dietary habits and geographical provenance. Hum. Microbiome J. 2018, 7-8, 1-9. [CrossRef]

21. Zhang, J.; Kobert, K.; Flouri, T.; Stamatakis, A. PEAR: A fast and accurate Illumina Paired-End reAd mergeR. Bioinformatics 2014, 30, 614-620. [CrossRef] [PubMed]

22. Bolger, A.M.; Lohse, M.; Usadel, B. Trimmomatic: A flexible trimmer for Illumina sequence data. Bioinformatics 2014, 30, 2114-2120. [CrossRef]

23. Caporaso, J.G.; Kuczynski, J.; Stombaugh, J.; Bittinger, K.; Bushman, F.D.; Costello, E.K.; Fierer, N.; Pena, A.G.; Goodrich, J.K.; Gordon, J.I.; et al. QIIME allows analysis of high-throughput community sequencing data. Nat. Methods 2010, 7, 335-336. [CrossRef] [PubMed]

24. DeSantis, T.Z.; Hugenholtz, P.; Larsen, N.; Rojas, M.; Brodie, E.L.; Keller, K.; Huber, T.; Dalevi, D.; Hu, P.; Andersen, G.L. Greengenes, a chimera-checked 16S rRNA gene database and workbench compatible with ARB. Appl. Environ. Microbiol. 2006, 72, 5069-5072. [CrossRef] [PubMed]

25. Wickham, H. ggplot2; Springer: New York, NY, USA, 2009; Volume 8, p. 213. [CrossRef] 
26. McMurdie, P.J.; Holmes, S. phyloseq: An R package for reproducible interactive analysis and graphics of microbiome census data. PLoS ONE 2013, 8, e61217. [CrossRef] [PubMed]

27. Chong, J.; Liu, P.; Zhou, G.; Xia, J. Using MicrobiomeAnalyst for comprehensive statistical, functional, and meta-analysis of microbiome data. Nat. Protoc. 2020, 15, 799-821. [CrossRef] [PubMed]

28. Segata, N.; Izard, J.; Waldron, L.; Gevers, D.; Miropolsky, L.; Garrett, W.S.; Huttenhower, C. Metagenomic biomarker discovery and explanation. Genome Biol. 2011, 12, R60. [CrossRef] [PubMed]

29. Zheng, P.; Li, Z.; Zhou, Z. Gut microbiome in type 1 diabetes: A comprehensive review. Diabetes Metab. Res. Rev. 2018, 34, e3043. [CrossRef]

30. Davis-Richardson, A.G.; Ardissone, A.N.; Dias, R.; Simell, V.; Leonard, M.T.; Kemppainen, K.M.; Drew, J.C.; Schatz, D.; Atkinson, M.A.; Kolaczkowski, B.; et al. Bacteroides dorei dominates gut microbiome prior to autoimmunity in Finnish children at high risk for type 1 diabetes. Front. Microbiol. 2014, 5, 678. [CrossRef]

31. De Goffau, M.C.; Fuentes, S.; van den Bogert, B.; Honkanen, H.; de Vos, W.M.; Welling, G.W.; Hyoty, H.; Harmsen, H.J. Aberrant gut microbiota composition at the onset of type 1 diabetes in young children. Diabetologia 2014, 57, 1569-1577. [CrossRef]

32. Meijnikman, A.S.; Gerdes, V.E.; Nieuwdorp, M.; Herrema, H. Evaluating Causality of Gut Microbiota in Obesity and Diabetes in Humans. Endocr. Rev. 2018, 39, 133-153. [CrossRef] [PubMed]

33. Knip, M.; Honkanen, J. Modulation of Type 1 Diabetes Risk by the Intestinal Microbiome. Curr. Diabetes Rep. 2017, 17, 105. [CrossRef]

34. Giongo, A.; Gano, K.A.; Crabb, D.B.; Mukherjee, N.; Novelo, L.L.; Casella, G.; Drew, J.C.; Ilonen, J.; Knip, M.; Hyoty, H.; et al. Toward defining the autoimmune microbiome for type 1 diabetes. ISME J. 2011, 5, 82-91. [CrossRef]

35. Tascini, G.; Berioli, M.G.; Cerquiglini, L.; Santi, E.; Mancini, G.; Rogari, F.; Toni, G.; Esposito, S. Carbohydrate Counting in Children and Adolescents with Type 1 Diabetes. Nutrients 2018, 10, 109. [CrossRef]

36. Hirsch, I.B. Type 1 diabetes mellitus and the use of flexible insulin regimens. Am. Fam. Physician 1999, 60, $2343-2352$.

37. Szypowska, A.; Schwandt, A.; Svensson, J.; Shalitin, S.; Cardona-Hernandez, R.; Forsander, G.; Sundberg, F.; De Beaufort, C.; Maahs, D.; Maffeis, C.; et al. Insulin pump therapy in children with type 1 diabetes: Analysis of data from the SWEET registry. Pediatr. Diabetes 2016, 17 (Suppl. S23), 38-45. [CrossRef]

38. Ziel, F.H.; Davidson, M.B. The role of glucosylated serum albumin in monitoring glycemic control in stable insulin-requiring diabetic out-patients. J. Clin. Endocrinol. Metab. 1987, 64, 269-273. [CrossRef]

39. Derrien, M.; Van Baarlen, P.; Hooiveld, G.; Norin, E.; Muller, M.; de Vos, W.M. Modulation of Mucosal Immune Response, Tolerance, and Proliferation in Mice Colonized by the Mucin-Degrader Akkermansia muciniphila. Front. Microbiol. 2011, 2, 166. [CrossRef]

40. Lukovac, S.; Belzer, C.; Pellis, L.; Keijser, B.J.; de Vos, W.M.; Montijn, R.C.; Roeselers, G. Differential modulation by Akkermansia muciniphila and Faecalibacterium prausnitzii of host peripheral lipid metabolism and histone acetylation in mouse gut organoids. mBio 2014, 5, e01438-14. [CrossRef]

41. Desai, M.S.; Seekatz, A.M.; Koropatkin, N.M.; Kamada, N.; Hickey, C.A.; Wolter, M.; Pudlo, N.A.; Kitamoto, S.; Terrapon, N.; Muller, A.; et al. A Dietary Fiber-Deprived Gut Microbiota Degrades the Colonic Mucus Barrier and Enhances Pathogen Susceptibility. Cell 2016, 167, 1339-1353.e1321. [CrossRef]

42. Reunanen, J.; Kainulainen, V.; Huuskonen, L.; Ottman, N.; Belzer, C.; Huhtinen, H.; de Vos, W.M.; Satokari, R. Akkermansia muciniphila Adheres to Enterocytes and Strengthens the Integrity of the Epithelial Cell Layer. Appl. Environ. Microbiol. 2015, 81, 3655-3662. [CrossRef]

43. Ottman, N.; Huuskonen, L.; Reunanen, J.; Boeren, S.; Klievink, J.; Smidt, H.; Belzer, C.; de Vos, W.M. Characterization of Outer Membrane Proteome of Akkermansia muciniphila Reveals Sets of Novel Proteins Exposed to the Human Intestine. Front. Microbiol. 2016, 7, 1157. [CrossRef]

44. Collado, M.C.; Derrien, M.; Isolauri, E.; de Vos, W.M.; Salminen, S. Intestinal integrity and Akkermansia muciniphila, a mucindegrading member of the intestinal microbiota present in infants, adults, and the elderly. Appl. Environ. Microbiol. 2007, 73, 7767-7770. [CrossRef] [PubMed]

45. Shin, N.R.; Lee, J.C.; Lee, H.Y.; Kim, M.S.; Whon, T.W.; Lee, M.S.; Bae, J.W. An increase in the Akkermansia spp. population induced by metformin treatment improves glucose homeostasis in diet-induced obese mice. Gut 2014, 63, 727-735. [CrossRef]

46. Everard, A.; Belzer, C.; Geurts, L.; Ouwerkerk, J.P.; Druart, C.; Bindels, L.B.; Guiot, Y.; Derrien, M.; Muccioli, G.G.; Delzenne, N.M.; et al. Cross-talk between Akkermansia muciniphila and intestinal epithelium controls diet-induced obesity. Proc. Natl. Acad. Sci. USA 2013, 110, 9066-9071. [CrossRef]

47. Li, F.; Wang, M.; Wang, J.; Li, R.; Zhang, Y. Alterations to the Gut Microbiota and Their Correlation With Inflammatory Factors in Chronic Kidney Disease. Front. Cell Infect. Microbiol. 2019, 9, 206. [CrossRef]

48. Brown, C.T.; Davis-Richardson, A.G.; Giongo, A.; Gano, K.A.; Crabb, D.B.; Mukherjee, N.; Casella, G.; Drew, J.C.; Ilonen, J.; Knip, M.; et al. Gut microbiome metagenomics analysis suggests a functional model for the development of autoimmunity for type 1 diabetes. PLoS ONE 2011, 6, e25792. [CrossRef] [PubMed]

49. Shin, J.; Noh, J.R.; Chang, D.H.; Kim, Y.H.; Kim, M.H.; Lee, E.S.; Cho, S.; Ku, B.J.; Rhee, M.S.; Kim, B.C.; et al. Elucidation of Akkermansia muciniphila Probiotic Traits Driven by Mucin Depletion. Front. Microbiol. 2019, 10, 1137. [CrossRef]

50. Li, J.; Lin, S.; Vanhoutte, P.M.; Woo, C.W.; Xu, A. Akkermansia Muciniphila Protects Against Atherosclerosis by Preventing Metabolic Endotoxemia-Induced Inflammation in Apoe-/- Mice. Circulation 2016, 133, 2434-2446. [CrossRef] 
51. Arumugam, M.; Raes, J.; Pelletier, E.; Le Paslier, D.; Yamada, T.; Mende, D.R.; Fernandes, G.R.; Tap, J.; Bruls, T.; Batto, J.M.; et al. Enterotypes of the human gut microbiome. Nature 2011, 473, 174-180. [CrossRef]

52. Dao, M.C.; Everard, A.; Aron-Wisnewsky, J.; Sokolovska, N.; Prifti, E.; Verger, E.O.; Kayser, B.D.; Levenez, F.; Chilloux, J.; Hoyles, L.; et al. Akkermansia muciniphila and improved metabolic health during a dietary intervention in obesity: Relationship with gut microbiome richness and ecology. Gut 2016, 65, 426-436. [CrossRef]

53. Benson, A.K.; Kelly, S.A.; Legge, R.; Ma, F.; Low, S.J.; Kim, J.; Zhang, M.; Oh, P.L.; Nehrenberg, D.; Hua, K.; et al. Individuality in gut microbiota composition is a complex polygenic trait shaped by multiple environmental and host genetic factors. Proc. Natl. Acad. Sci. USA 2010, 107, 18933-18938. [CrossRef] [PubMed]

54. McKnite, A.M.; Perez-Munoz, M.E.; Lu, L.; Williams, E.G.; Brewer, S.; Andreux, P.A.; Bastiaansen, J.W.; Wang, X.; Kachman, S.D.; Auwerx, J.; et al. Murine gut microbiota is defined by host genetics and modulates variation of metabolic traits. PLoS ONE 2012, 7, e39191. [CrossRef]

55. Henao-Mejia, J.; Elinav, E.; Jin, C.; Hao, L.; Mehal, W.Z.; Strowig, T.; Thaiss, C.A.; Kau, A.L.; Eisenbarth, S.C.; Jurczak, M.J.; et al. Inflammasome-mediated dysbiosis regulates progression of NAFLD and obesity. Nature 2012, 482, 179-185. [CrossRef]

56. Peng, J.; Narasimhan, S.; Marchesi, J.R.; Benson, A.; Wong, F.S.; Wen, L. Long term effect of gut microbiota transfer on diabetes development. J. Autoimmun. 2014, 53, 85-94. [CrossRef]

57. Fakhro, K.A.; Staudt, M.R.; Ramstetter, M.D.; Robay, A.; Malek, J.A.; Badii, R.; Al-Marri, A.A.; Abi Khalil, C.; Al-Shakaki, A.; Chidiac, O.; et al. The Qatar genome: A population-specific tool for precision medicine in the Middle East. Hum. Genome Var. 2016, 3, 16016. [CrossRef]

58. Fontana, A.; Panebianco, C.; Picchianti-Diamanti, A.; Lagana, B.; Cavalieri, D.; Potenza, A.; Pracella, R.; Binda, E.; Copetti, M.; Pazienza, V. Gut Microbiota Profiles Differ among Individuals Depending on Their Region of Origin: An Italian Pilot Study. Int. J. Environ. Res. Public Health 2019, 16, 4065. [CrossRef] [PubMed]

59. Turpin, W.; Espin-Garcia, O.; Xu, W.; Silverberg, M.S.; Kevans, D.; Smith, M.I.; Guttman, D.S.; Griffiths, A.; Panaccione, R.; Otley, A.; et al. Association of host genome with intestinal microbial composition in a large healthy cohort. Nat. Genet. 2016, 48, 1413-1417. [CrossRef]

60. Walter, J.; Ley, R. The human gut microbiome: Ecology and recent evolutionary changes. Annu. Rev. Microbiol. 2011, 65, 411-429. [CrossRef] [PubMed]

61. Mullaney, J.A.; Stephens, J.E.; Costello, M.E.; Fong, C.; Geeling, B.E.; Gavin, P.G.; Wright, C.M.; Spector, T.D.; Brown, M.A.; Hamilton-Williams, E.E. Type 1 diabetes susceptibility alleles are associated with distinct alterations in the gut microbiota. Microbiome 2018, 6, 35. [CrossRef]

62. Silverman, M.; Kua, L.; Tanca, A.; Pala, M.; Palomba, A.; Tanes, C.; Bittinger, K.; Uzzau, S.; Benoist, C.; Mathis, D. Protective major histocompatibility complex allele prevents type 1 diabetes by shaping the intestinal microbiota early in ontogeny. Proc. Natl. Acad. Sci. USA 2017, 114, 9671-9676. [CrossRef] [PubMed]

63. Zhang, X.S.; Li, J.; Krautkramer, K.A.; Badri, M.; Battaglia, T.; Borbet, T.C.; Koh, H.; Ng, S.; Sibley, R.A.; Li, Y.; et al. Antibioticinduced acceleration of type 1 diabetes alters maturation of innate intestinal immunity. Elife 2018, 7, e37816. [CrossRef]

64. Livanos, A.E.; Greiner, T.U.; Vangay, P.; Pathmasiri, W.; Stewart, D.; McRitchie, S.; Li, H.; Chung, J.; Sohn, J.; Kim, S.; et al. Antibiotic-mediated gut microbiome perturbation accelerates development of type 1 diabetes in mice. Nat. Microbiol. 2016, 1, 16140. [CrossRef] [PubMed]

65. Kemppainen, K.M.; Vehik, K.; Lynch, K.F.; Larsson, H.E.; Canepa, R.J.; Simell, V.; Koletzko, S.; Liu, E.; Simell, O.G.; Toppari, J.; et al. Association Between Early-Life Antibiotic Use and the Risk of Islet or Celiac Disease Autoimmunity. JAMA Pediatr. 2017, 171, 1217-1225. [CrossRef]

66. Rodrigues, R.R.; Greer, R.L.; Dong, X.; DSouza, K.N.; Gurung, M.; Wu, J.Y.; Morgun, A.; Shulzhenko, N. Antibiotic-Induced Alterations in Gut Microbiota Are Associated with Changes in Glucose Metabolism in Healthy Mice. Front. Microbiol. 2017, 8 , 2306. [CrossRef] 